\title{
Highly Automated Vehicles
}

\section{Dear Reader,}

Intelligent assistance systems are finding their way into more and more areas of our lives. For example, many car drivers now rely on automatic parking systems and adaptive cruise control. There is also a clear trend for more assistance systems in trucks, many of which now come as standard with an electronic stability program and a lane departure warning and emergency braking system. In future, a combination of camera and radar based systems and the use of vehicle data will provide more support for drivers and greater safety for all road users.

These developments are also having an impact in the world of agricultural engineering. In particular in the industrial nations, modern agriculture is a highly automated sector. This is one of the reasons why manufacturers are integrating more and more powerful functions into their machines. Because of the relatively low numbers of vehicles sold in the agricultural industry, the development costs for these systems are comparatively high. However, the Autosar standard, which is widely used in the automotive industry, can help in this respect. Until now it has not been possible to apply this standard to agricultural machinery, because it did not support Isobus. A new solution from ITK Engineering has brought together Autosar and Isobus. You can find out how this was achieved in our first cover story on page 6 .

The second cover story on page 16 highlights the added value offered by driver assistance systems and electric powertrains in the agricultural industry. The driving and assistance systems in the Innovation Tractor 2016 concept vehicle allow the tractor to manoeuvre semi- autonomously or with the help of mobile devices outside the cab. Here too, the goal is to improve the efficiency and safety of agricultural machinery.

Other manufacturers are following the same path. Case IH presented its cabless tractor at the Farm Progress show in the USA (see the title page). The vehicle is controlled using an interactive user interface. The onboard system automatically takes into account the width of the tractor's implements and plots the most efficient route, depending on the terrain, any obstructions and the other machines in use in the same field. The operator can monitor and correct the routes that the tractor takes from a desktop computer or a tablet.

The interesting question is: which of these systems and solutions will be successful? Ultimately, the customer will provide the answer.
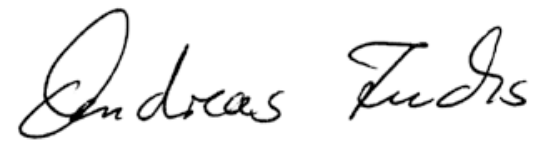

\section{Andreas Fuchs}

Chief Correspondent

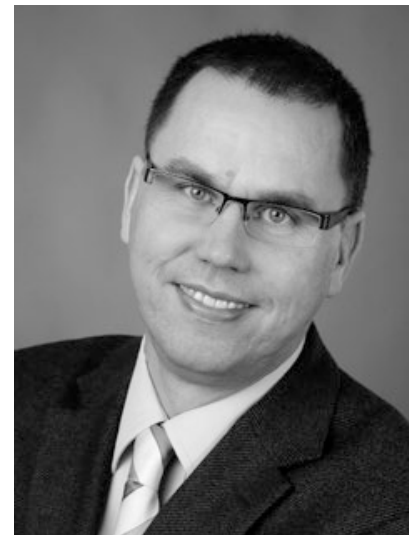

